\title{
GRADIENT ESTIMATE FOR EIGENFORMS OF HODGE LAPLACIAN
}

\author{
JiAPING WANG AND Linfeng ZHOU
}

\begin{abstract}
In this paper, we derive a gradient estimate for linear combinations of eigenforms of the Hodge Laplacian on a closed manifold. The estimate is given in terms of the dimension, volume, diameter and curvature bound of the manifold. As an application, we obtain directly a sharp estimate for the heat kernel of the Hodge Laplacian.
\end{abstract}

\section{Introduction}

Let $\left(M^{n}, g\right)$ be a compact oriented Riemannian manifold without boundary. The Hodge Laplacian $\Delta: A^{p}(M) \rightarrow A^{p}(M)$, acting on the space of smooth $p$-forms $A^{p}(M)$ on $M$, is defined by

$$
\Delta=-d \delta-\delta d
$$

Here, as usual, $d$ is the exterior differential operator and $\delta$ the adjoint of $d$ with respect to the $L^{2}$ inner product on $A^{p}(M)$. We denote the eigenvalues of $\Delta$ by $\left\{0 \leq \lambda_{1} \leq\right.$ $\left.\ldots \lambda_{k} \leq \ldots\right\}$ with a corresponding orthonormal basis of eigenforms $\left\{\phi_{i}\right\}_{i=1}^{\infty}$. We have the following estimate concerning the eigenforms.

Theorem 1.1. Let $\left(M^{n}, g\right)$ be a closed manifold with curvature bound $|R m| \leq K$. Then for any $b_{i} \in \mathbb{R}$ with $\sum_{i=1}^{k} b_{i}^{2} \leq 1$, the form $\omega=\sum_{i=1}^{k} b_{i} \phi_{i}$ satisfies the estimate

$$
|\nabla \omega|^{2}+\left(\lambda_{k}+1\right)|\omega|^{2} \leq c\left(\lambda_{k}+1\right)^{\frac{n}{2}+1}
$$

where $c=c(n, V, d, K)$ is an explicit constant depending on the dimension $n$, volume $V$, diameter $d$ and the curvature bound $K$.

We would like to emphasize that the estimate is valid for all finite linear combinations of the eigenforms, and it does not involve any covariant derivatives of the curvature tensor. Also, the exponent $\frac{n}{2}+1$ in $\lambda_{k}$ is sharp. This sharp exponent in turn leads to another one in $k$ for the lower bound of the eigenvalue $\lambda_{k} \geq c k^{-\frac{2}{n}}$ for all $k>b_{p}$, the $p$-th Betti number of $M$.

Our estimates can then be applied to analyze the heat kernel of $\Delta$. Combining with a result of Rumin [8], one has the following Sobolev inequality for $p$-forms.

Theorem 1.2. For an explicit constant $c=c(n, V, d, K)$,

$$
\left(\int_{M}|\omega-P(\omega)|^{\frac{2 n}{n-2}}\right)^{\frac{n-2}{n}} \leq c \int_{M}\left\{|d \omega|^{2}+|\delta \omega|^{2}\right\}
$$

Received by the editors September 21, 2011.

1991 Mathematics Subject Classification. 58J50.

Key words and phrases. Hodge Laplacian, gradient estimate, Sobolev inequality. 
for all smooth $p$-form $\omega$ on $M$, where $P(\omega)$ denotes the projection of $\omega$ on to the space of harmonic $p$-forms.

Another consequence is the following Hessian estimate for the eigenfunctions on $M$.

Corollary 1.3. Let $\left(M^{n}, g\right)$ be a closed manifold with curvature bound $|R m| \leq K$. Let $\phi_{1}, \phi_{2}, \cdots, \phi_{k}$ be orthonormal eigenfunctions of the scalar Laplacian with corresponding eigenvalues $0<\lambda_{1} \leq \lambda_{2} \leq \cdots \leq \lambda_{k}$. Then there exists a constant $c(K, d, V, n)$ such that

where $f=\sum_{i=1}^{k} b_{i} \phi_{i}$ and $\sum_{i=1}^{k} b_{i}^{2}=1$.

$$
|\nabla d f| \leq c \lambda_{k}^{\frac{n+4}{4}}
$$

Let us point out that the analysis of the Laplacian on a compact manifold is a classical subject. Numerous contributions have been made by various authors. While the results here are mostly known, we do hope our seemingly more direct treatment is of certain expository value.

As well-known, the gradient estimate method was successfully employed by Yau [11] to study harmonic functions on complete manifolds. The method was further developed by $\mathrm{Li}$ [4], and $\mathrm{Li}$ and Yau [6] to study eigenfunctions and eigenvalues. In particular, they have obtained a lower bound for the first non-zero eigenvalue of the scalar Laplacian in terms of the lower bound of Ricci curvature and the diameter of the manifold.

Our current work is very much motivated by and follows the ideas in a famous paper of $\mathrm{Li}$ [5], where he has obtained a lower bound for higher eigenvalues of the Hodge Laplacian. This is achieved through an estimate of the linear combinations of the eigenforms. The estimate involves the curvature operator lower bound and the Sobolev constant of the manifold, but not the curvature upper bound. However, the estimate there seems insufficient to provide a sharp exponent for the eigenvalue lower bounds alluded above. We would also like to point out that both E. Aubry's PhD thesis and the paper [10] by W. Ballmann, J. Brüning and G. Carron have already developed a gradient estimate for individual eigenforms.

The case of scalar Laplacian deserves special attention as it is of more common concern. So we will treat it separately in section 2. The result is a bit stronger in the sense it only involves the Ricci curvature lower bound in all the estimates. The approach is also more straightforward as it only relies on a direct application of the maximum principle.

The case of general Hodge Laplacian is handled in section 3. The proof now involves an iteration scheme as in [5].

Finally, we mention that the results here can be extended to the case of compact manifolds with boundary. For the ease of exposition, we omit the details here.

\section{Analysis of scalar Laplacian}

In this section, we will derive a variant version of the well-known gradient estimates of Li-Yau [6] concerning the eigenfunctions. As an application, we give direct proofs to some well-known results including a lower bound of the high eigenvalues, the existence of heat kernel and its long time decay estimate.

Let $\left(M^{n}, g\right)$ be a closed Riemannian manifold with diameter $d$, volume $V$, and Ricci curvature lower bound $-(n-1) K$, where $K \geq 0$ is a constant. Denote the eigenvalues 
of the Laplacian $\Delta$ by $0=\lambda_{0}<\lambda_{1} \leq \cdots \leq \lambda_{k} \leq \cdots$ with the corresponding eigenfunction $\phi_{i}, i=0,1,2, \cdots$, satisfying

$$
\Delta \phi_{i}=-\lambda_{i} \phi_{i}, \quad \int_{M} \phi_{i} \phi_{j}=\delta_{i j}
$$

For a given constant $c$, consider the function

$$
Q(x)=|\nabla \phi|^{2}+c \phi^{2},
$$

where $\phi=\sum_{i=1}^{k} b_{i} \phi_{i}$ with $b_{i} \in \mathbb{R}$ and $\sum_{i=1}^{k} b_{i}^{2}=1$. Obviously, the maximum value of $Q(x)$ over $M$ is a function of $b_{1}, \cdots, b_{k}$. This function in turn achieves its maximum at some point $a_{1}, \cdots, a_{k}$. Let $u=\sum_{i=1}^{k} a_{i} \phi_{i}$.

\section{Lemma 2.1.}

$$
|\nabla u|^{2}+c u^{2} \leq c \max _{M} u^{2}
$$

where $c=\lambda_{k}+(n-1) K$.

Proof. Define

$$
F\left(b_{1}, \ldots, b_{k}, x, \lambda\right)=Q(x)-\lambda\left(\sum_{i=1}^{k} b_{i}^{2}-1\right) .
$$

Then, subject to the constraint $\sum_{i=1}^{k} b_{i}^{2}=1, F$ achieves its maximum value at some point $\left(a_{1}, \cdots, a_{k}, x_{0}, \alpha\right)$. We now show

$$
|\nabla u|^{2}\left(x_{0}\right)+c u^{2}\left(x_{0}\right) \leq c \max _{M} u^{2}
$$

for $c>\lambda_{k}+(n-1) K$.

At the point $\left(a_{1}, \cdots, a_{k}, x_{0}, \alpha\right), F$ satisfies

$$
\left\{\begin{array}{l}
\nabla F\left(a_{1}, \cdots, a_{k}, x_{0}, \alpha\right)=0 \\
\Delta F\left(a_{1}, \cdots, a_{k}, x_{0}, \alpha\right) \leq 0 \\
\frac{\partial F}{\partial b_{i}}=0 \\
\sum_{i=1}^{k} a_{i}^{2}=1
\end{array}\right.
$$

From the third equation of (2.1), we have

$$
\sum_{j=1}^{k}\left(2 a_{j}\left\langle\nabla \phi_{i}, \nabla \phi_{j}\right\rangle+2 c a_{j} \phi_{i} \phi_{j}\right)-2 \alpha a_{i}=0 .
$$

After multiplying by $a_{i}$ and summing over $i$, one sees that

$$
\alpha=Q\left(u, x_{0}\right)=|\nabla u|^{2}\left(x_{0}\right)+c u^{2}\left(x_{0}\right) .
$$

Suppose now that

$$
|\nabla u|^{2}\left(x_{0}\right)+c u^{2}\left(x_{0}\right)>c \max _{M} u^{2} .
$$

Then

$$
\nabla u\left(x_{0}\right) \neq 0
$$

and one can choose an orthonormal frame $\left\{e_{1}, \ldots, e_{n}\right\}$ at $x_{0}$ so that

$$
\nabla u\left(x_{0}\right)=u_{1}\left(x_{0}\right) e_{1} .
$$


Now the first equation of $(2.1), \nabla F\left(a_{1}, \ldots, a_{k}, x_{0}, \alpha\right)=0$, becomes

$$
2 u_{1} u_{1 i}+2 c u u_{i}=0
$$

for $i=1, \ldots, n$. This in particular implies

$$
|\nabla \nabla u|^{2} \geq u_{11}^{2}=c^{2} u^{2} .
$$

On the other hand, at the maximum point $\left(a_{1}, \cdots, a_{k}, x_{0}, \alpha\right)$,

$$
\Delta F\left(a_{1}, \ldots, a_{k}, x_{0}, \alpha\right) \leq 0
$$

or

$$
\Delta|\nabla u|^{2}+c \Delta u^{2} \leq 0
$$

By the Bochner formula, it becomes

$$
|\nabla \nabla u|^{2}+\langle\nabla \Delta u, \nabla u\rangle+\operatorname{Ric}(\nabla u, \nabla u)+c u \Delta u+c|\nabla u|^{2} \leq 0 .
$$

In view of (2.2) and the lower bound of Ricci curvature, the above inequality reduces to

$$
c^{2} u^{2}+\langle\nabla \Delta u, \nabla u\rangle-(n-1) K|\nabla u|^{2}+c u \Delta u+c|\nabla u|^{2} \leq 0 .
$$

Note that

$$
\Delta u=-\sum_{i=1}^{k} \lambda_{i} a_{i} \phi_{i} .
$$

Therefore,

$$
\begin{aligned}
0 & \geq c^{2} u^{2}+(c-(n-1) K)|\nabla u|^{2}-\sum_{i, j=1}^{k} \lambda_{i} a_{i} a_{j}\left\langle\nabla \phi_{i}, \nabla \phi_{j}\right\rangle-\sum_{i, j=1}^{k} c \lambda_{i} a_{i} a_{j} \phi_{i} \phi_{j} \\
& \geq c^{2} u^{2}+(c-(n-1) K)|\nabla u|^{2}-\sum_{i=1}^{k} \lambda_{i} a_{i} \sum_{j=1}^{k}\left(a_{j}\left\langle\nabla \phi_{i}, \nabla \phi_{j}\right\rangle+c a_{j} \phi_{i} \phi_{j}\right) \\
& \geq c^{2} u^{2}+(c-(n-1) K)|\nabla u|^{2}-\sum_{i=1}^{k} \alpha \lambda_{i} a_{i}^{2} \\
& \geq c^{2} u^{2}+(c-(n-1) K)|\nabla u|^{2}-\alpha \lambda_{k} \\
& \geq c^{2} u^{2}+(c-(n-1) K)|\nabla u|^{2}-\lambda_{k}\left(|\nabla u|^{2}+c u^{2}\right) \\
& \geq c\left(c-\lambda_{k}\right) u^{2}+\left(c-(n-1) K-\lambda_{k}\right)|\nabla u|^{2} .
\end{aligned}
$$

This is an obvious contradiction if $c>(n-1) K+\lambda_{k}$. In other words,

$$
|\nabla u|^{2}\left(x_{0}\right)+c u^{2}\left(x_{0}\right) \leq c \max _{M} u^{2}
$$

for all $c>(n-1) K+\lambda_{k}$. The lemma follows by letting $c$ approach $\lambda_{k}+(n-1) K$.

As a consequence, we obtain a quick proof to the following well-known facts.

Theorem 2.2. There exists a constant $c(K, d, V, n)$ such that

$$
|\nabla \phi|^{2} \leq c \lambda_{k}^{\frac{n+2}{2}}, \quad \phi^{2} \leq c \lambda_{k}^{\frac{n}{2}}
$$


In particular,

$$
\left|\nabla \phi_{k}\right| \leq c \lambda_{k}^{\frac{n+2}{4}}, \quad\left|\phi_{k}\right| \leq c \lambda_{k}^{\frac{n}{4}} .
$$

(2) For all $k \geq 1$,

$$
\lambda_{k} \geq c^{-1} k^{\frac{2}{n}}
$$

(3) The function $H(x, y, t)$ given by

$$
H(x, y, t)=\frac{1}{V}+\sum_{k=1}^{\infty} e^{-\lambda_{k} t} \phi_{k}(x) \phi_{k}(y)
$$

is a heat kernel of M. Moreover,

$$
\left|H(x, y, t)-\frac{1}{V}\right| \leq c t^{-\frac{n}{2}}
$$

for all $t>0$.

(4) The following Sobolev inequality holds.

$$
\left(\int_{M}|f|^{\frac{2 n}{n-2}}\right)^{\frac{n-2}{n}} \leq c \int_{M}|\nabla f|^{2}
$$

for all smooth function $f$ on $M$ with $\int_{M} f=0$.

Proof. (1) Let $u$ be the function considered in the preceding lemma. Then we need only to prove the estimate for $u$. Choose point $p$ such that

$$
u^{2}(p)=\max _{M} u^{2}
$$

For $r>0$ and $x \in B_{p}\left(\frac{r}{\sqrt{\lambda_{k}+(n-1) K}}\right)$, using lemma 2.1, we conclude

$$
\begin{aligned}
u^{2}(p)-u^{2}(x) & \leq \max _{y \in M} 2|u|(y)|\nabla u|(y) d(x, p) \\
& \leq 2 u^{2}(p) \sqrt{\lambda_{k}+(n-1) K} \frac{r}{\sqrt{\lambda_{k}+(n-1) K}} \\
& \leq 2 r u^{2}(p) .
\end{aligned}
$$

Therefore,

$$
u^{2}(x) \geq(1-2 r) u^{2}(p)
$$

on $B_{p}\left(\frac{r}{\sqrt{\lambda_{k}+(n-1) K}}\right)$. Integrating with respect to $x$ over the ball yields

$$
1=\|u\|_{L^{2}(M)}^{2} \geq(1-2 r) u^{2}(p) \frac{V_{p}\left(\frac{r}{\sqrt{\lambda_{k}+(n-1) K}}\right)}{V_{p}(d)} V_{p}(d) .
$$

Choose $r$ such that

$$
4 r<1 \quad \text { and } \quad \frac{r}{\lambda_{1}+\sqrt{(n-1) K}}<d .
$$

Then by the Bishop volume comparison theorem we have

$$
1 \geq(1-2 r) u^{2}(p) \frac{c(K, d, V, n)}{\left(\lambda_{k}+(n-1) K\right)^{\frac{n}{2}}} .
$$


In other words,

$$
u^{2}(x) \leq u^{2}(p) \leq c(K, d, V, n) \lambda_{k}^{\frac{n}{2}},
$$

where we have used the fact that $\lambda_{k} \geq \lambda_{1} \geq c$ by [6]. Using lemma 2.1 again, we also conclude

$$
|\nabla u|^{2}(x) \leq\left(\lambda_{k}+(n-1) K\right) u^{2}(p) \leq c(K, d, V, n) \lambda_{k}^{\frac{n+2}{2}} .
$$

(2) For each $x \in M$, there exists an orthogonal matrix $\left(a_{i j}\right)_{k \times k}$ such that

$$
\nabla \psi_{j}(x)=0
$$

for $j=n+1, \cdots, k$, where $\psi_{j}=\sum_{i=1}^{k} a_{i j} \phi_{i}$.

From (1), it follows that

$$
\begin{aligned}
\sum_{i=1}^{k}\left|\nabla \phi_{i}\right|^{2}(x) & =\sum_{j=1}^{n}\left|\nabla \psi_{j}\right|^{2}(x) \\
& \leq n \max _{j}\left|\nabla \psi_{j}\right|^{2} \\
& \leq c_{1} \lambda_{k}^{\frac{n+2}{2}} .
\end{aligned}
$$

Integrating the inequality with respect to $x$, we conclude

$$
\lambda_{1}+\lambda_{2}+\cdots+\lambda_{k} \leq c_{2} \lambda_{k}^{\frac{n+2}{2}} .
$$

By an elementary induction argument, the inequality implies

$$
\lambda_{k} \geq c_{3} k^{2 / n}
$$

for all $k \geq 1$, where $c_{3}=\min \left\{\lambda_{1},\left(\frac{1}{c_{2}} \frac{n}{n+2}\right)^{\frac{n}{2}}\right\}$.

(3) In view of (1) and (2), it is straightforward to check the infinite series

$$
\frac{1}{V}+\sum_{k=1}^{\infty} e^{-\lambda_{k} t} \phi_{k}(x) \phi_{k}(y)
$$

converges uniformly in the $C^{1}$ sense for $x, y \in M$ and $t \geq c$ for any $c>0$. It is then easy to verify the limit is a heat kernel of $M$.

Since

$$
c^{-1} k^{\frac{2}{n}} \leq \lambda_{k} \leq c k^{\frac{2}{n}}
$$

one sees by (1) that

$$
\begin{aligned}
\left|H(x, y, t)-\frac{1}{V}\right| & \leq \sum_{k=1}^{\infty} e^{-\lambda_{k} t}\left|\phi_{k}\right|(x)\left|\phi_{k}\right|(y) \\
& \leq \sum_{k=1}^{\infty} c \lambda_{k}^{\frac{n}{2}} e^{-\lambda_{k} t} \\
& \leq c t^{-\frac{n}{2}} \int_{0}^{\infty} s^{\frac{n}{2}} e^{-s} d s \\
& \leq c t^{-\frac{n}{2}} .
\end{aligned}
$$

(4) follows from (3) by a result of Varopoulos [9]. 
We remark that both (2) and (3) were first proved by Cheng and Li [1] using the Sobolev inequality. Historically, the Sobolev inequality on manifolds was derived from the isoperimetric inequalities, which were established by Yau [12] and Croke [3].

\section{Gradient estimate for eigenforms}

Using the well-known Bochner-Weitzenbock formula, one can directly apply the proof in the previous section to the Hodge Laplacian acting on the smooth $p$-forms on $M$. However, the resulting estimates depend also on the bounds of the covariant derivative of the curvature tensor of $M$. It turns out this dependency is superfluous by adopting a different argument as demonstrated by E. Aubry in his PhD thesis and also by W. Ballmann, J. Brüning and G. Carron in [10]. In the following, we present a slightly refined version of their argument to suit our purpose.

We will use the moving frame notations. So for a $p$-form $\omega$ on $M$, under an orthonormal coframe $\left\{\omega_{1}, \cdots, \omega_{n}\right\}, \omega=a_{i_{1} \cdots i_{p}} \omega_{i_{p}} \wedge \cdots \wedge \omega_{i_{1}}$.

The Bochner-Weitzenbock formula says

$$
\Delta \omega=\Delta_{B} \omega-E(\omega),
$$

where

is the Bochner Laplacian and

$$
\Delta_{B} \omega=a_{i_{1} \cdots i_{p}, j j} \omega_{i_{p}} \wedge \cdots \wedge \omega_{i_{1}}
$$

$$
E(\omega)=R_{k_{\beta} i_{\beta} j_{\alpha} i_{\alpha}} a_{i_{1} \ldots k_{\beta} \ldots i_{p}} \omega_{i_{p}} \wedge \cdots \wedge \omega_{j_{\alpha}} \wedge \cdots \wedge \omega_{i_{1}}
$$

with $R_{i j k l}$ being the curvature tensor of $M$. Now,

$$
\Delta_{B}(\nabla \omega)=a_{i_{1} \ldots i_{p}, i j j} \omega_{i_{p}} \wedge \cdots \wedge \omega_{i_{1}} \otimes \omega_{i}
$$

and

Hence

$$
\nabla \Delta \omega=a_{i_{1} \ldots i_{p}, j j i} \omega_{i_{p}} \wedge \cdots \wedge \omega_{i_{1}} \otimes \omega_{i}-\nabla(E(\omega)) .
$$

$$
\Delta_{B}(\nabla \omega)-\nabla \Delta \omega=a_{I, i j j} \omega_{I} \otimes \omega_{i}-a_{I, j j i} \omega_{I} \otimes \omega_{i}+\nabla(E(\omega)) .
$$

By the Ricci identity, we have

$$
a_{I, i j j}-a_{I, j i j}=\left(R_{j_{\alpha} i_{\alpha} i j} a_{i_{1} \ldots j_{\alpha} \ldots i_{p}}\right)_{, j}
$$

and

$$
a_{I, j i j}-a_{I, j j i}=R_{j_{\alpha} i_{\alpha} i j} a_{i_{1} \ldots j_{\alpha} \ldots i_{p}, j}+R_{l j i j} a_{i_{1} \ldots i_{p}, l} .
$$

Thus we have the commutation formula

$$
\begin{aligned}
\Delta_{B}(\nabla \omega)-\nabla \Delta \omega= & R_{l i} a_{i_{1} \ldots i_{p}, l} \omega_{i_{p}} \wedge \cdots \wedge \omega_{i_{1}} \otimes \omega_{i} \\
& +R_{j_{\alpha} i_{\alpha} i j} a_{i_{1} \ldots j_{\alpha} \ldots i_{p}, j} \omega_{i_{p}} \wedge \cdots \wedge \omega_{i_{1}} \otimes \omega_{i} \\
& +\left(R_{j_{\alpha} i_{\alpha} i j} a_{i_{1} \ldots j_{\alpha} \ldots i_{p}}\right)_{, j} \omega_{i_{p}} \wedge \cdots \wedge \omega_{i_{1}} \otimes \omega_{i} \\
& +\nabla(E(\omega)) .
\end{aligned}
$$

Finally, we conclude

$$
\begin{aligned}
\left\langle\Delta_{B}(\nabla \omega)-\nabla \Delta \omega, \nabla \omega\right\rangle= & R_{l i} a_{i_{1} \ldots i_{p}, l} a_{i_{1} \ldots i_{p}, i} \\
& +R_{j_{\alpha} i_{\alpha} i j} a_{i_{1} \ldots j_{\alpha} \ldots i_{p}, j} a_{i_{1} \ldots i_{\alpha} \ldots i_{p}, i} \\
& +\left(R_{j_{\alpha} i_{\alpha} i j} a_{i_{1} \ldots j_{\alpha} \ldots i_{p}}\right)_{, j} a_{i_{1} \ldots i_{\alpha} \ldots i_{p}, i} \\
& +\langle\nabla(E(\omega)), \nabla \omega\rangle
\end{aligned}
$$


Note that these formulas and the following lemma have more or less been derived by Le Couturier and G. Robert in [2].

We now consider the function $f=|\nabla \omega|^{2}+A|\omega|^{2}$, where $A \geq 1$ is a fixed constant.

Lemma 3.1. Let $\left(M^{n}, g\right)$ be a closed Riemannian manifold with curvature operator $|R m| \leq K$. Then for $k \geq 1$,

$$
\int_{M} f^{k-1} \Delta f \geq 2 \int_{M}(\langle\nabla \Delta \omega, \nabla \omega\rangle+A\langle\Delta \omega, \omega\rangle) f^{k-1}-c k^{2} \int_{M} f^{k},
$$

where $c=2 n K(K+2)+18 K^{2}$.

Proof. Direct calculation gives

$$
\begin{aligned}
\Delta f= & \Delta\left(|\nabla \omega|^{2}+A|\omega|^{2}\right) \\
= & 2\left\langle\Delta_{B}(\nabla \omega), \nabla \omega\right\rangle+2|\nabla \nabla \omega|^{2} \\
& +2 A|\nabla \omega|^{2}+2 A\left\langle\Delta_{B} \omega, \omega\right\rangle \\
= & 2\langle\nabla \Delta \omega, \nabla \omega\rangle+2 A\langle\Delta \omega, \omega\rangle \\
& +2|\nabla \nabla \omega|^{2}+2 A|\nabla \omega|^{2}+2 A\langle E(\omega), \omega\rangle \\
& +2\left\langle\Delta_{B}(\nabla \omega)-\nabla \Delta \omega, \nabla \omega\right\rangle .
\end{aligned}
$$

Therefore,

$$
\begin{aligned}
\int_{M} f^{k-1} \Delta f= & 2 \int_{M}(\langle\nabla \Delta \omega, \nabla \omega\rangle+A\langle\Delta \omega, \omega\rangle) f^{k-1} \\
& +2 \int_{M}\left(|\nabla \nabla \omega|^{2}+A|\nabla \omega|^{2}\right) f^{k-1} \\
& +2 A \int_{M}\langle E(\omega), \omega\rangle f^{k-1} \\
& +2 \int_{M}\left\langle\Delta_{B}(\nabla \omega)-\nabla \Delta \omega, \nabla \omega\right\rangle f^{k-1}
\end{aligned}
$$

Since $|R m| \leq K$,

$$
2 A \int_{M}\langle E(\omega), \omega\rangle f^{k-1} \geq-2 K \int_{M} f^{k}
$$

Using (3.2), we have

$$
\begin{aligned}
2 \int_{M} & \left\langle\Delta_{B}(\nabla \omega)-\nabla \Delta \omega, \nabla \omega\right\rangle f^{k-1} \\
= & 2 \int_{M} R_{l i} a_{i_{1} \ldots i_{p}, l} a_{i_{1} \ldots i_{p}, i} f^{k-1} \\
& +2 \int_{M} R_{j_{\alpha} i_{\alpha} i j} a_{i_{1} \ldots j_{\alpha} \ldots i_{p}, j} a_{i_{1} \ldots i_{\alpha} \ldots i_{p}, i} f^{k-1} \\
& +2 \int_{M}\left(R_{j_{\alpha} i_{\alpha} i j} a_{i_{1} \ldots j_{\alpha} \ldots i_{p}}\right)_{, j} a_{i_{1} \ldots i_{\alpha} \ldots i_{p}, i} f^{k-1} \\
& +2 \int_{M}\langle\nabla(E(\omega)), \nabla \omega\rangle f^{k-1} .
\end{aligned}
$$


The first and second term of (3.6) obviously satisfy

$$
2 \int_{M} R_{l i} a_{i_{1} \ldots i_{p}, l} a_{i_{1} \ldots i_{p}, i} f^{k-1} \geq-2(n-1) K \int_{M} f^{k} .
$$

and

$$
\begin{aligned}
2 \int_{M} R_{j_{\alpha} i_{\alpha} i j} a_{i_{1} \ldots j_{\alpha} \ldots i_{p}, j} a_{i_{1} \ldots i_{\alpha} \ldots i_{p}, i} f^{k-1} & \geq-2 K \int_{M}|\nabla \omega|^{2} f^{k-1} \\
& \geq-2 K \int_{M} f^{k}
\end{aligned}
$$

For the third term of (3.6), after integration by parts, we have

$$
\begin{aligned}
2 \int_{M} & \left(R_{j_{\alpha} i_{\alpha} i j} a_{i_{1} \ldots j_{\alpha} \ldots i_{p}}\right)_{, j} a_{i_{1} \ldots i_{\alpha} \ldots i_{p}, i} f^{k-1} \\
= & -2 \int_{M} R_{j_{\alpha} i_{\alpha} i j} a_{i_{1} \ldots j_{\alpha} \ldots i_{p}} a_{i_{1} \ldots i_{\alpha} \ldots i_{p}, i j} f^{k-1} \\
& -2(k-1) \int_{M} R_{j_{\alpha} i_{\alpha} i j} a_{i_{1} \ldots j_{\alpha} \ldots i_{p}} a_{i_{1} \ldots i_{\alpha} \ldots i_{p}, i} f^{k-2} f_{j} \\
\geq & -2 K \int_{M}|\omega||\nabla \nabla \omega| f^{k-1} \\
& -2(k-1) K \int_{M}|\omega||\nabla \omega| f^{k-2}|\nabla f| \\
\geq & -2 K^{2} \int_{M} f^{k}-\frac{1}{2} \int_{M}|\nabla \nabla \omega|^{2} f^{k-1} \\
& -8 k^{2} K^{2} \int_{M} f^{k}-\frac{1}{2} \int_{M}\left(|\nabla \nabla \omega|^{2}+A|\nabla \omega|^{2}\right) f^{k-1},
\end{aligned}
$$

where we have used the fact that

$$
|\omega||\nabla \omega| \leq f
$$

and

$$
\begin{aligned}
|\nabla f| & \leq 2|\nabla \omega||\nabla \nabla \omega|+2 A|\omega||\nabla \omega| \\
& \leq 4 k K\left(|\nabla \omega|^{2}+A|\omega|^{2}\right)+\frac{1}{4 k K}\left(|\nabla \nabla \omega|^{2}+A|\nabla \omega|^{2}\right) .
\end{aligned}
$$

Applying integration of parts to the last term of (3.6), we get

$$
\begin{aligned}
2 \int_{M} & \langle\nabla(E(\omega)), \nabla \omega\rangle f^{k-1} \\
\geq & -2 \int_{M}\left\langle E(\omega), \Delta_{B} \omega\right\rangle f^{k-1} \\
& \quad-2(k-1) \int_{M}|E(\omega)||\nabla \omega| f^{k-2}|\nabla f|
\end{aligned}
$$




$$
\begin{aligned}
\geq & -2 \sqrt{n} K \int_{M}|\omega||\nabla \nabla \omega| f^{k-1} \\
& -2(k-1) K \int_{M}|\omega||\nabla \omega| f^{k-2}|\nabla f| \\
\geq & -2 n K^{2} \int_{M} f^{k}-\frac{1}{2} \int_{M}|\nabla \nabla \omega|^{2} f^{k-1} \\
& -8 k^{2} K^{2} \int_{M} f^{k}-\frac{1}{2} \int_{M}\left(|\nabla \nabla \omega|^{2}+A|\nabla \omega|^{2}\right) f^{k-1},
\end{aligned}
$$

where we have used (3.10) in the last step.

Putting (3.7), (3.8), (3.9) and (3.11) into (3.6), we conclude

$$
\begin{aligned}
2 \int_{M} & \left\langle\Delta_{B}(\nabla \omega)-\nabla \Delta \omega, \nabla \omega\right\rangle f^{k-1} \\
\geq & -\left(2 n K(K+1)+18 k^{2} K^{2}\right) \int_{M} f^{k} \\
& -2 \int_{M}\left(|\nabla \nabla \omega|^{2}+A|\nabla \omega|^{2}\right) f^{k-1} .
\end{aligned}
$$

Plugging (3.5) and (3.12) into (3.4), we arrived at

$$
\begin{aligned}
\int_{M} f^{k-1} \Delta f \geq & 2 \int_{M}(\langle\nabla \Delta \omega, \nabla \omega\rangle+A\langle\Delta \omega, \omega\rangle) f^{k-1} \\
& -\left(2 n K(K+2)+18 k^{2} K^{2}\right) \int_{M} f^{k}
\end{aligned}
$$

The lemma is proved.

We now prove a gradient estimate concerning the linear combinations of eigenforms.

Theorem 3.2. Let $\left(M^{n}, g\right)$ be a closed manifold with curvature bound $|R m| \leq K$. Let $\phi_{1}, \phi_{2}, \cdots, \phi_{l}$ be orthonormal eigenforms of the Hodge Laplacian $\Delta$ acting on the $p$-forms with corresponding eigenvalues $0 \leq \lambda_{1} \leq \lambda_{2} \leq \cdots \leq \lambda_{l}$. Then for any $b_{i} \in \mathbb{R}$ with $\sum_{i=1}^{l} b_{i}^{2} \leq 1$, the form $\omega=\sum_{i=1}^{l} b_{i} \phi_{i}$ satisfies the estimate

$$
|\nabla \omega|^{2}+A|\omega|^{2} \leq c\left(\lambda_{l}+K+1\right)^{\frac{n}{2}+1},
$$

where $A=\lambda_{l}+K+1$, and $c=c(n, V, d, K)$ is a constant.

Proof. For each $k \geq 1$, let

$$
I_{k}=\max \int_{M} f^{2 k},
$$

where $f=|\nabla \omega|^{2}+A|\omega|^{2}$ and the maximum is taken over all $\omega=\sum_{i=1}^{l} b_{i} \phi_{i}$ with $b_{i} \in \mathbb{R}$ and $\sum_{i=1}^{l} b_{i}^{2} \leq 1$.

Note that for $\omega=\sum_{i=1}^{l} b_{i} \phi_{i}$ with $\sum_{i=1}^{l} b_{i}^{2} \leq 1$,

$$
\Delta \omega=-\sum_{i=1}^{l} \lambda_{i} b_{i} \phi_{i}=-\lambda_{l} \sum_{i=1}^{l} a_{i} \phi_{i}
$$


where $a_{i}=\lambda_{i} \lambda_{l}^{-1} b_{i}, i=1, \cdots, l$. Obviously,

$$
\sum_{i=1}^{l} a_{i}^{2} \leq 1
$$

So if we denote $\eta=\sum_{i=1}^{l} a_{i} \phi_{i}$, then

$$
\begin{aligned}
& \int_{M}(\langle\nabla \Delta \omega, \nabla \omega\rangle+A\langle\Delta \omega, \omega\rangle) f^{2 k-1} \\
& \quad \geq-\int_{M}\left(|\nabla \Delta \omega|^{2}+A|\Delta \omega|^{2}\right)^{\frac{1}{2}} f^{2 k-\frac{1}{2}} \\
& \quad \geq-\lambda_{l}\left(\int_{M}\left(|\nabla \eta|^{2}+A|\eta|^{2}\right)^{2 k}\right)^{\frac{1}{4 k}}\left(\int_{M} f^{2 k}\right)^{\frac{4 k-1}{4 k}} \\
& \quad \geq-\lambda_{l} I_{k} .
\end{aligned}
$$

Combining with lemma 3.1, we have the estimate

$$
\int_{M} f^{2 k-1} \Delta f \geq-\left(2 \lambda_{l}+c_{1} k^{2}\right) I_{k},
$$

where $c_{1}=8 n K(K+2)+72 K^{2}$.

On the other hand

$$
\int_{M} f^{2 k-1} \Delta f=-\frac{2 k-1}{k^{2}} \int_{M}\left|\nabla f^{k}\right|^{2} .
$$

Applying the Sobolev inequality

$$
\left(\int_{M}|u|^{2 \beta}\right)^{\frac{1}{\beta}} \leq C_{s}\left(\int_{M}|\nabla u|^{2}+\int_{M} u^{2}\right),
$$

where $\beta=\frac{n}{n-2}$, to $u=f^{k}$, we get

$$
\left(\int_{M} f^{2 k \beta}\right)^{\frac{1}{\beta}} \leq C_{s}\left(\int_{M}\left|\nabla f^{k}\right|^{2}+\int_{M} f^{2 k}\right) .
$$

Combining (3.13), (3.14) and (3.15), we get

$$
\left(\int_{M} f^{2 k \beta}\right)^{\frac{1}{\beta}} \leq C_{s} k\left(\lambda_{l}+c_{1} k^{2}\right) I_{k}
$$

Since this is true for all $\omega$, we may maximize the left hand side over $\omega$ and conclude

$$
\left(I_{\beta k}\right)^{\frac{1}{\beta k}} \leq\left(C_{s} k\left(\lambda_{l}+c_{1} k^{2}\right)\right)^{\frac{1}{k}}\left(I_{k}\right)^{\frac{1}{k}}
$$

for all $k \geq 1$.

Let $k=\beta^{i}, i=0,1,2, \cdots$ and iterate the preceding inequality. Then,

$$
\begin{aligned}
\lim _{i \rightarrow \infty}\left(I_{\beta^{i}}\right)^{\frac{1}{\beta^{i}}} & \leq \prod_{i=0}^{\infty}\left(C_{s} \beta^{i}\left(\lambda_{l}+c_{1} \beta^{2 i}\right)\right)^{\frac{1}{\beta^{i}}} I_{1} \\
& \leq c_{2}\left(\lambda_{l}+1\right)^{\frac{n}{2}} I_{1},
\end{aligned}
$$


where $c_{2}=c_{2}(n, d, V, K)$ is a constant. In other words,

$$
\begin{aligned}
& \max _{\omega} \max _{x \in M}\left(|\nabla \omega|^{2}+A|\omega|^{2}\right)^{2}(x) \\
& \leq c_{2}\left(\lambda_{l}+1\right)^{\frac{n}{2}} \max _{\omega} \int_{M}\left(|\nabla \omega|^{2}+A|\omega|^{2}\right)^{2} \\
& \leq c_{2}\left(\lambda_{l}+1\right)^{\frac{n}{2}} \max _{\omega} \max _{x \in M}\left(|\nabla \omega|^{2}+A|\omega|^{2}\right)(x) \max _{\omega} \int_{M}\left(|\nabla \omega|^{2}+A|\omega|^{2}\right) .
\end{aligned}
$$

Hence,

$$
\max _{\omega} \max _{x \in M}\left(|\nabla \omega|^{2}+A|\omega|^{2}\right)(x) \leq c_{2}\left(\lambda_{l}+1\right)^{\frac{n}{2}} \max _{\omega} \int_{M}\left(|\nabla \omega|^{2}+A|\omega|^{2}\right) .
$$

However,

$$
\begin{aligned}
\int_{M}\left(|\nabla \omega|^{2}+A|\omega|^{2}\right) & =-\int_{M}\langle\Delta \omega, \omega\rangle-\int_{M}\langle E(\omega), \omega\rangle+c \int_{M}|\omega|^{2} \\
& \leq \lambda_{l}+K+A
\end{aligned}
$$

Therefore,

$$
\max _{\omega} \max _{x \in M}\left(|\nabla \omega|^{2}+A|\omega|^{2}\right)(x) \leq c_{2}\left(\lambda_{l}+1\right)^{\frac{n}{2}}\left(\lambda_{l}+K+A\right) .
$$

The theorem is proved.

As in section 2, we can draw the following conclusions from theorem 3.2.

Theorem 3.3. Let $\left(M^{n}, g\right)$ be a closed manifold with curvature bound $|R m| \leq K$. Let $0 \leq \lambda_{1} \leq \lambda_{2} \leq \cdots \leq \lambda_{k} \leq \cdots$ be all the eigenvalues of the Hodge Laplacian $\Delta$ acting on the $p$-forms, and $\phi_{1}, \phi_{2}, \cdots, \phi_{k}, \cdots$ the corresponding orthonormal eigenforms. Then there exists a constant $c(K, d, V, n)$ such that

(1) $\left|\nabla \phi_{k}\right| \leq c\left(\lambda_{k}+1\right)^{\frac{n+2}{4}}$ and $\left|\phi_{k}\right| \leq c\left(\lambda_{k}+1\right)^{\frac{n}{4}}$.

(2) For all $k>b_{p}$, the $p$-th Betti number of $M$,

$$
\lambda_{k} \geq c^{-1} k^{\frac{2}{n}} .
$$

(3) The tensor $H_{p}(x, y, t)$ given by

$$
H_{p}(x, y, t)=\sum_{k=1}^{\infty} e^{-\lambda_{k} t} \phi_{k}(x) \otimes \phi_{k}(y)
$$

is a heat kernel of $\Delta$. Moreover,

$$
\left|H_{p}(x, y, t)-\sum_{k=1}^{b_{p}} \phi_{k}(x) \otimes \phi_{k}(y)\right| \leq c t^{-\frac{n}{2}}
$$

for all $t>0$.

(4) The following Sobolev inequality holds.

$$
\left(\int_{M}|\omega-P(\omega)|^{\frac{2 n}{n-2}}\right)^{\frac{n-2}{n}} \leq c \int_{M}\left\{|d \omega|^{2}+|\delta \omega|^{2}\right\}
$$

for all smooth p-form $\omega$ on $M$, where $P(\omega)$ denotes the projection of $\omega$ on to the space of harmonic p-forms. 
Proof. (1) is obvious by theorem 3.2. Using theorem 3.2, (2) follows as in the proof of (2) in theorem 2.2, where we now use a result of T. Mantuano [7] that $\lambda_{b_{p}+1} \geq$ $c(n, V, d, K)$. For (3), the proof is the same as (3) in theorem 2.2. Finally, (4) follows from (3) by Theorem 1.2 in [8].

We also have the following corollary concerning the eigenfunctions.

Corollary 3.4. Let $\left(M^{n}, g\right)$ be a closed manifold with curvature bound $|R m| \leq K$. Let $\phi_{1}, \phi_{2}, \cdots, \phi_{k}$ be orthonormal eigenfunctions of the scalar Laplacian with corresponding eigenvalues $0<\lambda_{1} \leq \lambda_{2} \leq \cdots \leq \lambda_{k}$. Then there exists a constant $c(K, d, V, n)$ such that

where $\phi=\sum_{i=1}^{k} b_{i} \phi_{i}$ and $\sum_{i=1}^{k} b_{i}^{2}=1$.

$$
|\nabla d \phi| \leq c \lambda_{k}^{\frac{n+4}{4}}
$$

Proof. Note that $d \phi_{i}$ is an eigenform for the Hodge Laplacian acting on the one forms. Now the corollary follows by applying theorem 3.2 to the one form setting with $d \phi_{i}$ normalized to have unit length in the $L^{2}$ sense.

As a final remark, it is possible to make explicit of all the constants in our arguments. In particular, we could spell out their dependency on the geometric quantities $d, V$ and $K$.

\section{Acknowledgments}

We would like to thank Gilles Carron for his insightful comments which lead to various improvement to the paper. Part of the paper was written while the second author was visiting the School of Mathematics at the University of Minnesota. He deeply appreciates its hospitality. He would also like to thank Gang Liu for his helpful comments. The first author is partially supported by NSF grant no. DMS-1105799 and the second author by NSFC grant no. 11271132.

\section{References}

[1] S. Y. Cheng and P. Li, Heat kernel estimates and lower bound of eigenvalues, Comment. Math. Helv. 56(3) (1981), 327-338.

[2] M. L. Couturier and G. Robert, $L^{p}$-pinching and the geometry of compact Riemannian manifolds, Comment. Math. Helv. 69(2) (1994), 249-271.

[3] C. Croke, Some isoperimetric inequalities and eigenvalue estimates, Ann. Sci. École Norm. Sup. (4) 13(4) (1980), 419-435.

[4] P. Li, A lower bound for the first eigenvalue of the Laplacian on a compact manifold, Indiana Univ. Math. J. 28(6) (1979), 1013-1019.

[5] P. Li, On the Sobolev constant and the p-spectrum of a compact Riemannian manifold, Ann. Sci. École Norm. Sup. (4) 13(4) (1980), 451-468.

[6] P. Li and S. T. Yau, Estimates of eigenvalues of a compact Riemannian manifold., in Geometry of the Laplace operator, 205-239, Proc. Sympos. Pure Math., XXXVI, Amer. Math. Soc., Providence, R. I. (1980).

[7] T. Mantuano, Discretization of Riemannian manifolds applied to the Hodge Laplacian, Amer. J. Math. 130(6) (2008), 1477-1508.

[8] M. Rumin, Spectral density and Sobolev inequalities for pure and mixed states, Geom. Funct. Anal. 20(3) (2010), 817-844.

[9] N. Varopoulos, Hardy-Littlewood theory for semigroups, J. Funct. Anal. 63 (1985) 240-260.

[10] W. Ballmann, J. Brüning and G. Carron, Eigenvalues and holonomy, Int. Math. Res. Not. 12 (2003) 657-665. 
[11] S. T. Yau, Harmonic functions on complete Riemannian manifolds, Comm. Pure Appl. Math. 28 (1975) 201-228.

[12] S. T. Yau, Isoperimetric constants and the first eigenvalue of a compact Riemannian manifold, Ann. Sci. École Norm. Sup. (4) 8(4) (1975), 487-507.

School of Mathematics, University of Minnesota, Minneapolis, MN 55455, USA

E-mail address: jiaping@math.umn.edu

Department of Mathematics, East China Normal University, Shanghai 200241, China

E-mail address: Ifzhou@math.ecnu.edu.cn 